\title{
Fuerza de agarre como predictor de composición corporal en estudiantes universitarias
}

\section{Handgrip strength as a predictor of body composition in female college students}

\begin{abstract}
RESUMEN
La relevancia que tiene la medición de la fuerza de agarre (FA) se ha incrementado en los últimos años como marcador del estado nutricional, por su relación con la morbilidad y mortalidad y por su creciente aplicación clínica y epidemiológica. Estudios de FA en adultos jóvenes sanos, revelan que se asocia positivamente con variables antropométricas y de composición corporal, mismas que se vincularían con la promoción de la salud en esta población. El objetivo de este estudio fue determinar la correlación entre la FA con variables de antropometría, composición corporal medida por bioimpedancia eléctrica y con la realización de ejercicio, para evaluar su utilidad como indicador del estado nutricional en jóvenes universitarias. Los resultados mostraron que en las estudiantes universitarias la FA se correlacionó positiva y significativamente con variables antropométricas (talla), y de composición corporal (masa muscular). Los profesionales de la salud requieren promover entre este grupo de población la reducción de los factores de riesgo como la baja fuerza muscular a través de la promoción de un estilo de vida activo y en específico de la realización de ejercicio físico de fuerza.

Palabras clave: Composición corporal; Dinamometría; Fuerza de agarre; Manual; Universitarias.
\end{abstract}

Ana Gabriela Palos Lucio ${ }^{1 *}$, María Judith Rios Lugo', Claudia Inés Victoria Campos ${ }^{1}$, Olivia González Acevedo ${ }^{1}$, Mónica Lucia Acebo Martínez', Dario Gaytán Hernández', Argenis Giraldo García², Gloria C. Deossa Restrepo².

*Dirigir correspondencia a: Ana Gabriela Palos Lucio, Facultad de Enfermería y Nutrición. Universidad Autónoma de San Luis Potosí. San Luis Potosí, México. Av. Niño Artillero \#130, Zona Universitaria. CP 78240. Email: gabriela.palos@uaslp.mx

Este trabajo fue recibido el 23 de septiembre de 2019. Aceptado con modificaciones: 27 de febrero de 2020. Aceptado para ser publicado: 23 de abril de 2020.

\section{ABSTRACT}

The relevance of measuring handgrip strength (HGS) has increased in recent years as a marker of nutritional status, due to its relationship with morbidity and mortality and its increasing clinical and epidemiological application. HGS studies in healthy young adults reveal that it is positively associated with anthropometric and body composition variables, the same indicators used for health promotion among young university students. The objective of this study was to determine the correlation between the HGS with anthropometry variables, body composition measured by electrical bioimpedance and with exercise performance, to evaluate its usefulness as an indicator of nutritional status in college students. The results showed that the HGS of female college students was positively and significantly correlated with anthropometric variables (height), and body composition (muscle mass). Among this population group, health professionals need to promote the reduction of risk factors such as low muscle strength through the promotion of an active lifestyle and, specifically, strength exercises. Key words: College students; Body composition; Dynamometer; Handgrip Strength.

\section{INTRODUCCIÓN}

En la prevención de riesgos cardiometabólicos y de mortalidad en diferentes poblaciones, la masa muscular (MM) ha sido considerada como un componente significativo de salud, y un factor modificable independientemente de la edad y condición clínica. Una medida clínica factible para cuantificar la función muscular es a través de la valoración de la fuerza 
muscular $(\mathrm{FM})^{1}$. Entre los adultos jóvenes, una mayor FM se ha asociado con perfiles de menor riesgo cardiometabólico como menores concentraciones de proteína C-reactiva e interleucina $6^{2,3}$, incluso con un efecto protector independiente y específico de mortalidad por cáncer e hipertensión ${ }^{4}$ y con la reducción en el riesgo de padecer diabetes tipo $2^{5}$. Hay evidencia científica de que la baja FM es un predictor significativo de ganancia de peso en un plazo de 20 años en adultos, lo que sugiere que la FM podría ayudar a atenuar la ganancia de peso relacionada con la edad y contribuir a la prevención de obesidad ${ }^{6}$. Específicamente, entre los estudiantes universitarios se ha evidenciado una relación entre la menor FM con obesidad, riesgo de síndrome metabólico y riesgo cardiovascular?

Desde los 80's se ha desarrollado un método no invasivo, eficiente de utilizar, portátil, confiable y de bajo costo que evalúa la función muscular, ésta es la fuerza de agarre (FA) o también llamada fuerza prensil manual o fuerza de empuñadura. La FA se correlaciona con la proporción de pérdida proteica y muestra cambios precoces de deprivación de nutrimentos si se compara con indicadores de composición corporal'. La relevancia que tiene la medición de la FA se ha incrementado en los últimos años como marcador del estado nutricional, por su relación con la morbilidad y mortalidad ${ }^{8}$ y por su creciente aplicación clínica y epidemiológica, como parte del diagnóstico de sarcopenia, propuesta por el Grupo de Trabajo Europeo sobre la Sarcopenia en Personas de edad Avanzada (EWGSOP) 9 .

En los últimos años, la FA ha sido utilizada para estimar datos de salud y más recientemente, de rendimiento físico ${ }^{10,11,12,13}$. Está influenciada por la hora del día en la que es medida, la edad, la condición nutricional y de salud, el género, la actividad física, la motivación y el tamaño de los sujetos; a su vez, se asocia con factores que intervienen en la calidad del músculo como determinantes ambientales y genéticos ${ }^{14}$.

Estudios de FA en adultos jóvenes sanos, revelan que se asocia positivamente con variables antropométricas y de composición corporal, mismas que se verían influenciadas por condiciones que promuevan la salud de las jóvenes universitarias ${ }^{14}$. El objetivo de este estudio fue determinar la correlación entre la FA con variables de antropometría, composición corporal medida por bioimpedancia eléctrica y con la realización de ejercicio, para evaluar su utilidad como indicador del estado nutricional en jóvenes universitarias.

\section{METODOLOGÍA \\ Diseño del estudio y muestra de la población}

El presente estudio forma parte del macroproyecto: "Cambios en estado nutricional, hábitos de alimentación y estilos de vida, en estudiantes de Nutrición y Dietética de Colombia y México: Estudio Multicéntrico". Tiene un diseño transversal descriptivo, en el que se evaluaron 62 jóvenes universitarias estudiantes de $1^{\circ}$ a $10^{\circ}$ semestre de la Licenciatura de Nutrición de la Facultad de Enfermería y Nutrición de la Universidad Autónoma de San Luis Potosí (UASLP), inscritas en el ciclo escolar 2016-2017. La muestra de estudio fue calculada con la fórmula para proporciones, con base en un error máximo permisible del $3,9 \%$ y un nivel de confianza del $95 \%$, donde los parámetros p y q se consideraron con valor de 0,5 debido a que no se tienen estudios anteriores; la muestra se seleccionó de manera aleatoria.

Entre los criterios de inclusión se consideró que fueran aparentemente sanas, sin presencia de enfermedades que comprometieran su estado nutricional, como autoinmunes o metabólicas, leucemia, cáncer, o insuficiencia renal; que no se encontraran en estado de gestación o lactancia y que no fueran deportistas de alto rendimiento, ni vegetarianas.

\section{Mediciones antropométricas, de composición corporal, de fuerza de agarre y de actividad física}

Se les solicitó a las estudiantes presentarse bajo ciertas condiciones antes de realizar las mediciones antropométricas y de composición corporal, con el fin de lograr una medición estable, y evitar que la resistencia del cuerpo se modificara por factores externos. Se les pidió asistir en ayuno, sin haberse sometido a una sauna, no haber realizado ejercicio y no haber ingerido alcohol, todo esto $12 \mathrm{~h}$ previas a la medición; $y$ haber evacuado anticipadamente ${ }^{15}$.

La evaluación antropométrica la realizó personal capacitado y estandarizado en la técnica descrita en el protocolo internacional para la valoración antropométrica de la Sociedad Internacional para el Avance de la Cineantropometría (ISAK, por sus siglas en inglés $)^{16}$. Se midió la estatura con un estadiómetro portátil marca SECA 206 (sensibilidad 0,1cm) misma que se registró en $\mathrm{cm}$. La circunferencia de cintura se determinó con cinta metálica marca Lufkin (sensibilidad 0,1 cm).

El peso y la composición corporal se evaluaron a través de una báscula digital con bioimpedancia marca Tanita (modelo BC-568, Tanita Corp. Tokyo, Japan). Este equipo es octo-polar (4 electrodos en las manos y 4 en los pies), el sujeto debe colocarse en posición de bipedestación y considera la estatura, el peso, sexo y actividad física del sujeto antes de hacer la valoración. Durante la medición se vigiló la correcta posición de los electrodos, se retiraron elementos metálicos, mientras que las jóvenes se encontraban descalzas y en ropa ligera. Dado que es imposible calcular el músculo esquelético total, la bioimpedancia realiza una medición de los grandes grupos musculares como brazos y piernas para proveer una estimación de la masa músculo-esquelética total basándose en cálculos derivados del análisis de regresión con las variables talla, edad, peso, y género ${ }^{17}$. La estimación del gasto metabólico basal es por medio de una fórmula desarrollada por Tanita ${ }^{\circledR}$ que trabaja bajo un análisis de regresión múltiple utilizando el valor de la masa libre de grasa de la bioimpedancia, y destaca por su alto nivel de eficiencia entre los individuos con diferente composición corporal ${ }^{18}$.

La fuerza de agarre se valoró en ambas manos con un dinamómetro estándar ajustable digital marca Takei (Takei Scientific Instruments Co., Ltd., Japan) en posición de pie, las estudiantes colocaban el dinamómetro en su mano a un costado de su cuerpo, en dirección hacia abajo. Se les pidió que ejecutaran su máxima fuerza en el dinamómetro. El máximo valor se reportó en kilos. Entre cada medición se le dio a la estudiante un período de descanso de 1 minuto, para evitar la fatiga. 
Tanto las mediciones de antropometría como las de fuerza de agarre, se hicieron por duplicado y se utilizó el promedio para el análisis estadístico.

La información sobre el ejercicio que realizaban las estudiantes se obtuvo de los resultados del Cuestionario sobre Conducta Alimentaria y Estilo de Vida que se les aplicó a las estudiantes como parte del Estudio Multicéntrico. Dicho cuestionario se componía de 8 apartados: 1. Datos generales; 2. Antecedentes familiares y personales patológicos; 3 . Hábitos de alimentación (frecuencia de consumo de alimentos; preferencias y rechazos alimentarios; compra, preparación y métodos de cocción de alimentos); 4 . Consumo de sustancias nocivas para la salud; 5 . Ejercicio y sedentarismo; 6 . Cambios en la alimentación; 7. Compras de alimentos; y 8. Percepción corporal. Para efectos del presente estudio, se analizaron los resultados del apartado $n^{\circ} 5$. Ejercicio y sedentarismo, en el que se indagaba acerca del tipo de ejercicio que realizaba la estudiante a través de la pregunta ¿cuál es el tipo de ejercicio que realizas (selecciona la que realizas con más frecuencia)?, las opciones de respuesta incorporaban ejercicios aeróbicos como caminar, bailar, andar en bicicleta, trotar o nadar y ejercicios de fuerza. Se averiguó sobre la duración del ejercicio, por medio de la pregunta ¿cuál es la duración del ejercicio que realizas?, las opciones de respuesta eran tres que incluían los rangos de $30 \mathrm{~min}$ a $1 \mathrm{~h}, 2$ a $3 \mathrm{~h}$ y más de $3 \mathrm{~h}$. La última pregunta fue sobre la frecuencia semanal en la que hacía ejercicio, la pregunta fue ¿cuántas veces a la semana realizas ejercicio?, las opciones de respuesta eran 1 vez, 2 veces, 3 veces, más de 3 veces, o no realiza. Sobre sedentarismo, el apartado incluía la pregunta ¿cuánto tiempo pasas frente a la pantalla (video juegos, computador, TV, otros)?, las opciones de respuesta fueron más de 3 horas, 4-6 h, 7-9 h y más de $9 \mathrm{~h}$.

\section{Definición de variables}

El diagnóstico de riesgo cardiovascular se definió a partir de los puntos de corte de la circunferencia de la cintura recomendados por la Secretaria de Salud de México $(\geq 90$ para hombres $y \geq 80$ para mujeres) ${ }^{19}$. El índice de masa corporal (IMC) se calculó al dividir el peso $(\mathrm{kg})$ entre la estatura al cuadrado $(\mathrm{m})$ y se caracterizó de acuerdo a los criterios de la Organización Mundial de la Salud (OMS): bajo peso (BMI $£ 18.5 \mathrm{~kg} / \mathrm{m}^{2}$ ), peso normal (IMC entre 18.5 y $24.9 \mathrm{~kg} / \mathrm{m}^{2}$ ), sobrepeso (IMC entre 25 y $\left.24.9 \mathrm{~kg} / \mathrm{m}^{2}\right)$ y obesidad $\left(\mathrm{IMC} \geq 30 \mathrm{~kg} / \mathrm{m}^{2}\right)^{20}$.

En cuanto a la fuerza de agarre en manos, se utilizaron tablas de referencia en sujetos sanos por género y edad de una población de Brasi $^{21}$ y de Teruel, España ${ }^{22}$, ambas validadas y expresadas en percentiles.

Los puntos de corte para emitir un diagnóstico acerca de la grasa corporal $(\%)$, masa muscular $(\mathrm{kg})$, grasa visceral, masa ósea, agua corporal y gasto metabólico basal fueron los establecidos por el aparato de bioimpedancia utilizado para hacer las mediciones de composición corporal en este estudio ${ }^{23}$.

\section{Consideraciones éticas}

Este proyecto fue aprobado por el Comité de Bioética de Odontología de la Universidad de Antioquia, en Medellín,
Colombia, mediante concepto No 17 del 2016, según Acta No 06 del 01 de agosto de 2016. Se obtuvo la firma del consentimiento informado de cada una de las estudiantes participantes en el estudio.

\section{Análisis estadístico}

Los datos se analizaron usando el paquete estadístico PASW Statistics 18. Se aplicaron herramientas descriptivas directamente a las mediciones y estadística inferencial específicamente la correlación lineal de Pearson, para evaluar las correlaciones de la fuerza de agarre con variables de composición corporal y realización de actividad física. Se realizaron análisis de varianza de una vía para comparar la fuerza media entre categorías de IMC y el tipo de ejercicio, considerando en todos los casos un nivel de confianza de $95 \%$.

\section{RESULTADOS}

La población de estudio estuvo conformada por 62 mujeres universitarias con una edad comprendida entre los 18 a 24 años, cuya ocupación fue para la mayoría de las participantes (98\%) estudiar de tiempo completo.

En la tabla 3 se observa que en el 95\% de los casos, las estudiantes se ubicaban en la clasificación de peso normal de acuerdo al IMC (IMC=20,7-22,6), y sin riesgo cardiovascular según la circunferencia de cintura. Sobre la composición corporal, los valores de los límites inferior y superior de grasa corporal de las estudiantes las ubicó, según la referencia, en la categoría de sobrepeso y obesidad (36\%-39\%); en cuanto a la masa muscular, el $95 \%$ fue clasificada en rango bajo según la edad y de grasa visceral en un rango saludable; por otra parte, la masa ósea de las estudiantes se encontró dentro de lo adecuado para su peso e como los valores del agua corporal total. Sobre la fuerza de agarre en mano derecha y mano izquierda, el 95\% de las estudiantes se encontraron entre el percentil 30 (P30) y percentil 50 (P50) para ambas manos según la tabla 1 y por arriba del percentil 75 (P75) y del P50 en la mano derecha e izquierda, respectivamente, de acuerdo con la tabla 2.

$\mathrm{Al}$ relacionar la fuerza de agarre en ambas manos con variables de antropometría y composición corporal, los hallazgos indicaron que la fuerza en mano derecha y la fuerza en mano izquierda se correlacionaron positivamente con la talla y la masa muscular $(p<0,001)$ es decir, mientras la fuerza de la mano aumenta, la talla y la masa muscular aumentaban también (Tabla 4).

Del total de las estudiantes y según las categorías del IMC, $16,1 \%$ se encontraron en bajo peso, $72,6 \%$ en peso normal, y $11,3 \%$ en sobrepeso. Al respecto, se observó una tendencia a que la fuerza de agarre aumente en la mano derecha y en la mano izquierda de acuerdo con las categorías de IMC, ya que las medias de la fuerza de agarre de la mano izquierda fueron $22,5 \mathrm{~kg}, 24,0 \mathrm{~kg}$ y $24,3 \mathrm{~kg}$, y para la fuerza de agarre en mano derecha fueron $24,9 \mathrm{~kg}$, 26,3 kg y 26,0 kg para el bajo peso, normal y sobrepeso, respectivamente. No obstante, las diferencias no fueron estadísticamente significativas al comparar las medias de la fuerza de agarre en ambas manos entre las categorías del IMC (Tabla 5). 
Con respecto a las variables de realización de ejercicio, se identificó que de acuerdo al tipo de ejercicio que hacían, la FA izquierda era mayor en aquellas estudiantes que hacían ejercicios en equipo; y que la FA derecha era mayor en las que realizaban ejercicios aeróbicos, no obstante, las diferencias no fueron estadísticamente significativas $(p>0,05)$. En cuanto a la frecuencia de realización de ejercicio, y la duración del mismo, las diferencias en FA izquierda y derecha, tampoco fueron significativas ( $p>0,05$ ); a pesar de esto se observó una tendencia de menor FA en aquellas que no realizaban ejercicio. En relación al tiempo frente a una pantalla al día y la FA, no se observaron diferencias significativas ( $>>0,05)$ (Tabla 6).

Tabla 1. Valores de la FA de población adulta de Río de Janeiro, Brasil.

\begin{tabular}{|c|c|c|c|c|c|c|c|c|c|c|}
\hline \multirow{2}{*}{$\begin{array}{l}\text { Mujeres } \\
\text { Edad (años) }\end{array}$} & \multicolumn{5}{|c|}{ Mano derecha $(\mathbf{k g})$} & \multicolumn{5}{|c|}{ Mano izquierda (kg) } \\
\hline & P10 & P30 & P50 & P70 & P90 & P10 & P30 & P50 & P70 & P90 \\
\hline $20-29$ & 19.5 & 23.8 & 27.4 & 30.0 & 34.0 & 18.6 & 22.3 & 25.8 & 28.4 & 31.8 \\
\hline
\end{tabular}

Tabla 2. Valores de la FA de población adulta de Teruel, España.

\begin{tabular}{|c|c|c|c|c|c|c|c|c|c|c|c|c|c|c|}
\hline \multirow{2}{*}{$\begin{array}{l}\text { Mujeres } \\
\text { Edad (años) }\end{array}$} & \multicolumn{7}{|c|}{ Mano derecha (kg) } & \multicolumn{7}{|c|}{ Mano izquierda (kg) } \\
\hline & P5 & P10 & P25 & P50 & P75 & P90 & P95 & P5 & P10 & P25 & P50 & P75 & P90 & P95 \\
\hline $20-29$ & 13.4 & 14.8 & 18.0 & 20.0 & 24.0 & 27.0 & 28.4 & 12.0 & 13.0 & 16.0 & 19.0 & 24.0 & 26.0 & 27.6 \\
\hline
\end{tabular}

Tabla 3. Características de las estudiantes de la Licenciatura de Nutrición.

\begin{tabular}{|c|c|c|c|c|c|c|}
\hline \multicolumn{7}{|c|}{ I.C. 95\% } \\
\hline Variable & Media & DE & Límite inferior & Límite superior & Mín. & Máx. \\
\hline Edad (años) & 20.2 & 1.7 & 19.6 & 20.7 & 18.0 & 24.0 \\
\hline \multicolumn{7}{|c|}{ Antropometría } \\
\hline Peso prom. (kg) & 57.2 & 9.6 & 54.3 & 60.1 & 41.9 & 84.5 \\
\hline Talla prom. (cm) & 162.2 & 5.0 & 160.7 & 163.7 & 151.6 & 177.3 \\
\hline CC. prom. (cm) & 72.0 & 8.3 & 69.5 & 74.5 & 58.3 & 101.2 \\
\hline $\operatorname{IMC}\left(\mathrm{kg} / \mathrm{m}^{2}\right)$ & 21.7 & 3.1 & 20.7 & 22.6 & 16.7 & 29.7 \\
\hline \multicolumn{7}{|c|}{ Composición corporal por BIA } \\
\hline $\mathrm{MM}(\mathrm{kg})$ & 27.8 & 6.8 & 25.8 & 29.9 & 14.8 & 44.7 \\
\hline GC (\%) & 38.6 & 3.4 & 37.6 & 39.6 & 32.1 & 47.6 \\
\hline GV & 1.8 & 1.3 & 1.4 & 2.2 & 1.0 & 6.0 \\
\hline $\mathrm{MO}(\mathrm{kg})$ & 3.8 & 5.7 & 2.0 & 5.5 & 1.7 & 24.0 \\
\hline AC (\%) & 53.3 & 4.5 & 51.9 & 54.7 & 42.9 & 62.6 \\
\hline GMB (kcal) & 2100 & 240 & 2023 & 2176 & 1689 & 2903 \\
\hline FA MD (kg) & 25.3 & 3.4 & 24.2 & 26.3 & 19.3 & 31.2 \\
\hline FA MI (kg) & 23.1 & 4.2 & 21.9 & 24.4 & 15.0 & 35.2 \\
\hline
\end{tabular}


Tabla 4. Correlaciones entre la edad, variables de antropometría y composición corporal, IMC y gasto metabólico basal con fuerza de agarre de las estudiantes de la Licenciatura en Nutrición.

Fuerza de agarre MD

Coeficiente
de correlación

0.22

0.070
Fuerza de agarre MI

Coeficiente
de correlación

0.15

0.210

Antropometría

$\begin{array}{lr}\text { Peso prom. }(\mathrm{kg}) & 0.17 \\ \text { Talla prom. }(\mathrm{cm}) & 0.32 \\ \text { Cint. prom. }(\mathrm{cm}) & 0.09 \\ \text { PC total }(\mathrm{mm}) & -0.08 \\ \mathrm{IMC}\left(\mathrm{kg} / \mathrm{m}^{2}\right) & 0.04\end{array}$

Edad

0.04
0.170
$<0.001$
0.480
0.514
0.750

$\begin{array}{rr}0.17 & 0.170 \\ 0.32 & <0.001 \\ 0.09 & 0.480 \\ 0.08 & 0.514 \\ 0.04 & 0.750\end{array}$

Valor de $\mathrm{p}^{*}$

\section{Composición corporal por BIA}

$\begin{array}{lrrrr}\text { MM }(\mathrm{kg}) & 0.41 & <0.001 & 0.39 & <0.001 \\ \text { GC }(\%) & -0.09 & 0.480 & 0.06 & 0.640 \\ \text { MO } & -0.05 & 0.684 & -0.07 & 0.602 \\ \text { GV } & 0.05 & 0.705 & 0.12 & 0.369 \\ \text { AC }(\%) & 0.09 & 0.494 & -0.01 & 0.965 \\ \text { GMB (Kcal) } & 0.19 & 0.140 & 0.16 & 0.210\end{array}$

MD: mano derecha. MI: mano izquierda. Peso prom: peso promedio. Talla prom.: talla promedio. Cint. prom.: cintura promedio. PC total: suma total de pliegues cutáneos. IMC: Índice de Masa Corporal. BIA: bioimpedancia eléctrica. MM: masa muscular. GC: grasa corporal. MO: masa ósea. GV: gasa visceral. AC: agua corporal total. GMB: gasto metabólico basal.

*Correlación lineal de Pearson.

Tabla 5. Diferencia de medias de la fuerza de agarre entre las categorías de IMC de las estudiantes de la Licenciatura en Nutrición.

\begin{tabular}{|c|c|c|c|c|c|c|}
\hline & IMC (i) & IMC (j) & Media i* & Media j* & $\begin{array}{c}\text { Diferencia de } \\
\text { medias }(\mathrm{i}-\mathrm{j})\end{array}$ & Valor de $\mathbf{p}^{+}$ \\
\hline \multirow[t]{6}{*}{ Fuerza de agarre MI } & \multirow[t]{2}{*}{ Bajo peso } & Normal & 22.5 & 24.0 & -1.5 & 0.9 \\
\hline & & Sobrepeso & 22.5 & 24.3 & -1.8 & 1.0 \\
\hline & \multirow[t]{2}{*}{ Normal } & Bajo peso & 24.0 & 22.5 & -1.8 & 0.9 \\
\hline & & Sobrepeso & 24.0 & 24.3 & -0.3 & 1.0 \\
\hline & \multirow[t]{2}{*}{ Sobrepeso } & Bajo peso & 24.3 & 22.5 & 1.8 & 1.0 \\
\hline & & (j) Normal & 24.3 & 24.0 & 0.3 & 1.0 \\
\hline \multirow[t]{6}{*}{ Fuerza de agarre MD } & \multirow[t]{2}{*}{ Bajo peso } & (i) Normal & 24.9 & 26.3 & -1.4 & 0.9 \\
\hline & & (j) Sobrepeso & 24.9 & 26.0 & -1.1 & 1.0 \\
\hline & \multirow[t]{2}{*}{ Normal } & (i) Bajo peso & 26.3 & 24.9 & -1.1 & 0.9 \\
\hline & & (j) Sobrepeso & 26.3 & 26.0 & 0.3 & 1.0 \\
\hline & \multirow[t]{2}{*}{ Sobrepeso } & (i) Bajo peso & 26.0 & 24.9 & 1.1 & 1.0 \\
\hline & & (j) Normal & 26.0 & 26.3 & -0.3 & 1.0 \\
\hline
\end{tabular}

MI: mano izquierda. MD: mano derecha. *i y j son símbolos que se utilizaron para indicar la comparación de las medias de las diferentes categorías del IMC. *ANOVA de una vía. 
Tabla 6. Tipo de ejercicio y fuerza de agarre de las estudiantes de la Licenciatura de Nutrición.

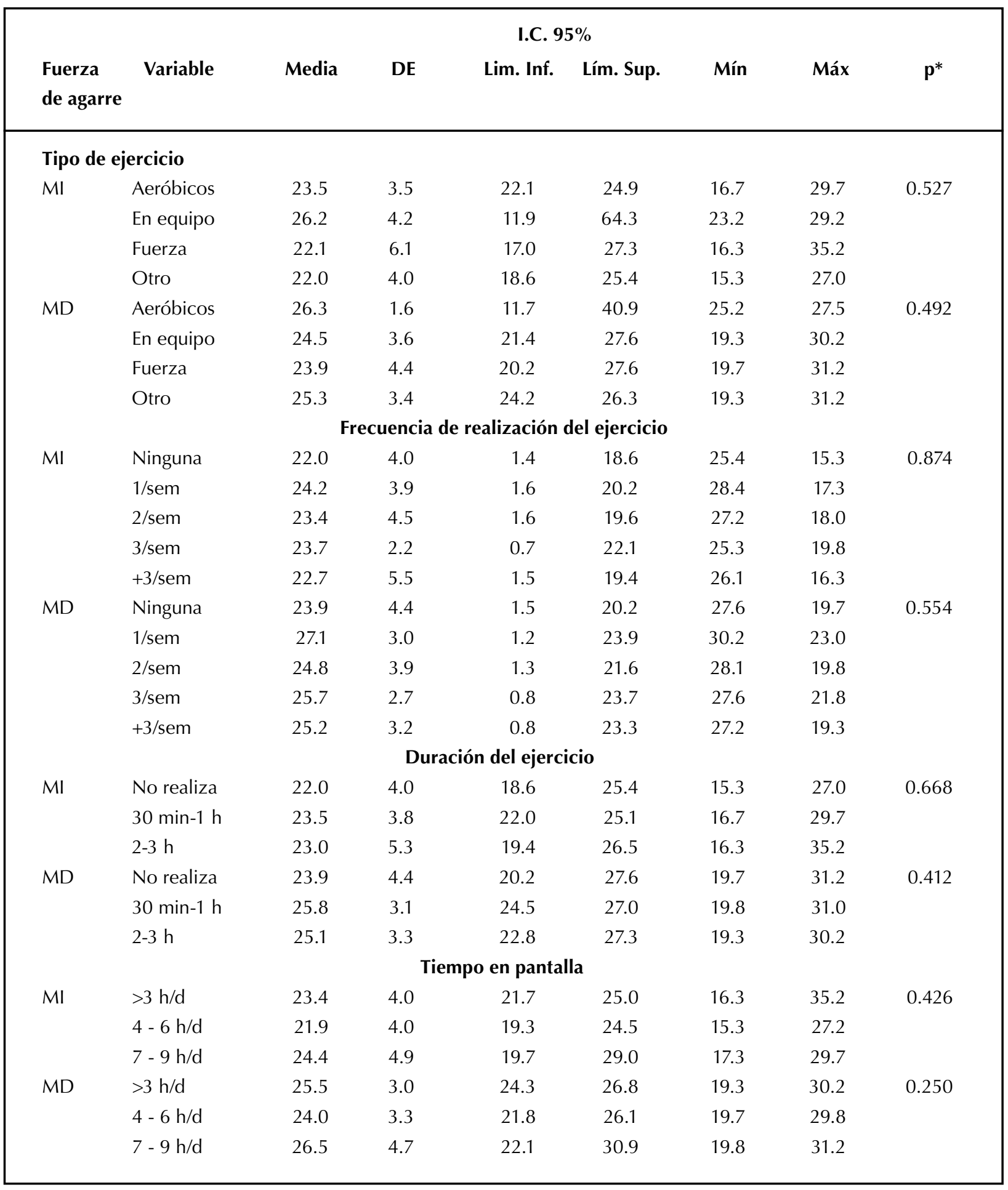

MI: mano izquierda. MD: mano derecha.

+ANOVA de una vía. 


\section{DISCUSIÓN}

La medición de la FA a través de la dinamometría se ha utilizado en los últimos años como indicador del estado nutricional pues permite evaluar de forma eficiente la fuerza de todo el cuerpo; por ende, se ha convertido en un componente de la evaluación nutricional. En este estudio se describen los datos obtenidos de la FA de un grupo de mujeres universitarias y se comparan con variables antropométricas, de composición corporal, con el IMC, con variables de realización de ejercicio y con el tiempo frente a una pantalla asociado a sedentarismo, con la finalidad de evaluar su grado de correlación y examinar su utilidad en la evaluación nutricional. Las mujeres universitarias que fueron evaluadas presentaron una correlación significativa positiva entre la FA y las variables antropométricas (talla), y de composición corporal (masa muscular).

Se han documentado relaciones significativas entre la máxima FA dominante y variables de antropometría. Diversos estudios han encontrado que la fuerza de agarre en sujetos sanos se correlaciona positivamente con la estatura, incluso con el peso ${ }^{24,25}$. Hallazgos similares a los nuestros se evidencian en el estudio de Rodríguez García et a ${ }^{26}$, en el que se exploró la relación entre la FA y factores antropométricos en una población mexicana adulta, concluyendo que la talla es el factor con la más fuerte correlación para la FA. Una posible explicación a esta correlación positiva pudiera ser el resultado de varios factores; los adultos con las mayores tallas, tienen regularmente brazos más largos que la mayoría, en consecuencia poseen una mayor palanca en el brazo para generar fuerza lo que resulta en una eficiente cantidad de fuerza aplicada.

Las mediciones de la FA, FM, y la masa muscular son una forma efectiva de evaluar la capacidad funcional y de salud física de un individuo, y se consideran factores modificables que protegen contra factores de riesgo metabólicos. En nuestro estudio, la FA de las jóvenes universitarias se encontró por arriba del p50 de acuerdo a la edad y sexo en concordancia con dos referencias con población adulta Latinoamericana $^{21,22}$. Si bien, los resultados de este estudio muestran que la población tenía una adecuada FA, es necesario identificar de forma temprana estos indicadores para así promover el mantenimiento de un estilo de vida saludable por medio de ejercicio físico y dieta saludable, lo que posibilita que las mujeres jóvenes universitarias preserven su masa muscular, fuerza y funcionalidad en esta etapa de la vida ${ }^{3,27}$. De igual manera, los resultados contribuyen a fomentar la FM como indicador de salud en los sistemas de vigilancia epidemiológica, justificándose claramente durante la etapa universitaria debido a que investigaciones en jóvenes universitarias señalan que una baja FM se relaciona con perfiles metabólicos pobres, obesidad, y dolores músculo esqueléticos, mismos que fomentarían resultados poco favorables en salud en su vida posterior ${ }^{7,28}$.

Son pocas las investigaciones que han intentado explicar la relación entre el IMC y la FA, la relación sigue siendo poco clara. Mientras algunos autores han evidenciado correlaciones positivas y significativas, algunos otros no ${ }^{29,30}$. En nuestro estudio correlacionamos estas dos variables y no encontramos diferencias significativas entre los grupos, esto pudiera deberse a que se ha demostrado que el género es un factor peculiar que afecta la fuerza de agarre e incluso el IMC. En este trabajo participaron únicamente estudiantes del género femenino, lo cual pudo afectar la diferencia de la fuerza de agarre en las categorías de IMC, como lo han explicado otros estudios, y referido como determinante crítico de la FA ${ }^{24,31}$. A su vez, se ha estudiado que el IMC no discrimina la composición corporal con exactitud, por lo que los resultados de esta investigación lo corroboran al relacionarse únicamente la talla y la masa muscular con la FA.

Aunque en diferentes estudios se ha propuesto que la realización de ejercicio es una variable que puede modificar la fuerza de agarre en manos, en este estudio esta relación no fue estadísticamente significativa. Esto podría deberse a que la mayoría de las jóvenes realizaban ejercicios aeróbicos; la evidencia sugiere que los entrenamientos de fuerza mejoran la FM en corto plazo ${ }^{32}$.

Entre las limitaciones de este estudio se considera el hecho de desconocer cuál era la mano dominante de las estudiantes para poder valorar con mayor certeza los resultados con las variables de realización de ejercicio. Se contempla también que las mediciones de la FA se realizaron en diferentes horarios, por lo que la influencia de la hora del día sobre ésta no se controló. Es probable que se requiera una muestra mayor para encontrar diferencias entre las categorías de IMC y la FA, pues ésta se reduce al estratificar a la población de estudio en los tres subgrupos de categorías de IMC. De acuerdo al tipo de estudio que se trata, un efecto causal entre las variables es difícil de identificar.

\section{CONCLUSIONES}

En este trabajo se encontraron relaciones significativas entre la FA de un grupo de mujeres jóvenes universitarias y variables antropométricas (talla) y de composición corporal (masa muscular). Los profesionales de la salud requieren promover entre este grupo de población la reducción de los factores de riesgo como la baja FM, a través del fomento de un estilo de vida activo, en específico de la realización de ejercicio físico de fuerza. Lo anterior, contribuiría a que variables de composición corporal en esta población puedan ubicarse en rangos de normalidad y por ende prevenir que la baja FM y la poca función muscular, se constituyan como un futuro problema de salud pública en este grupo. La medición de la FA es prometedora como un indicador del estado nutricional en diferentes grupos de edad, estados fisiológicos o enfermedades, por lo que deben realizarse más estudios al respecto.

\section{BIBLIOGRAFÍA}

1. Gómez-Londoño C, González-Correa G-C. Manual pressure force and correlation with anthropometric and physical condition in college students. Biosalud. 2012; 11: 11-19. 
2. Artero EG, Lee DC, Lavie Cl, et al. Effects of muscular strength on cardiovascular risk factors and prognosis. I Cardiopulm Rehabil Prev. 2012; 32: 351-358.

3. Garcia-Hermoso A, Tordecilla-Sanders A, Correa-Bautista $J E$, et al. Handgrip strength attenuates the adverse effects of overweight on cardiometabolic risk factors among collegiate students but not in individuals with higher fat levels. Sci Rep. 2019; 9: 1-8.

4. Artero EG, Lee DC, Ruiz IR, et al. A prospective study of muscular strength and all-cause mortality in men with hypertension. I Am Coll Cardiol. 2011; 57: 1831-1837.

5. Tarp I, Stole AP, Blond K, Grontved A. Cardiorespiratory fitness, muscular strength and risk of type 2 diabetes: a systematic review and meta-analysis. Diabetologia. 2019; 62: 1129-1142.

6. Jackson AW, Lee DC, Sui X, et al. Muscular strength is inversely related to prevalence and incidence of obesity in adult men. Obesity. 2010; 18: 1988-1995.

7. Garcia-Hermoso A, Correa-Bautista JE, Izquierdo $M$, et al. Ideal cardiovascular health, handgrip strength, and muscle mass among college students: The FUPRECOL Adults Study. J Strength Cond Res. 2019; 33: 747-754.

8. Gale CR, Martyn CN, Cooper C, Sayer AA. Grip strength, body composition, and mortality. Int J Epidemiol. 2007; 36: 228-235.

9. Cruz-Jentoft AJ, Bahat G, Bauer J, et al. Sarcopenia: revised European consensus on definition and diagnosis. Age Ageing. 2019; 48: 16-31.

10. McGrath RP, Kraemer WI, Al Snih S, Peterson MD. Handgrip Strength and Health in Aging Adults. Sports Medicine. 2018; 48: 1993-2000.

11. Peterson MD, Duchowny $K$, Meng $Q$, Wang $Y$, Chen $X$, Zhao $Y$. Low normalized grip strength is a biomarker for cardiometabolic disease and physical disabilities among U.S. and Chinese adults. I Gerontol A Biol Sci Med Sci. 2017; 7: 1525-1531.

12. Mainous AG, Tanner RJ, Anton SD, JA. Grip strength as a marker of hypertension and diabetes in healthy weight adults. Am J Prev Med. 2015; 49: 850-858.

13. Gubelmann $C$, Vollenweider $P$, Marques-Vidal P. Association of grip strength with cardiovascular risk markers. Eur J Prev Cardiol. 2017; 24: 514-521

14. Musa TH, Li W, Xiaoshan L, Guo Y, Wenjuan Y, Xuan Y, et al. Association of normative values of grip strength with anthropometric variables among students, in Jiangsu Province. Homo. 2018; 69: 70-76.

15. Tanita Technical Bulletin: Body Composition Measurement Repeatability. United States of America: Tanita Corporation of America Inc.; 2013 [accesed July 19th, 2019]. Availabe from: https://www.tanita.com/en/technicalbulletin/

16. Esparza-Ros F, Vaquero-Cristóbal R, Marfell-Jones $M$. International Protocol for Antropometric Measures. UCAM Catolic University of Murcia., Murcia, España, 2019.

17. Prentice AM. BIA Technology for assesing muscle mass. An introductory guide. Japan: Tanita Corporation; 2011.

18. Tanita Technical Bulletin: Regression Formula for Basal Metabolic Rate (BMR). United States of America: Tanita
Corporation of America Inc.; 2013 [accesed July 21, 2019] . Availabe from: https://www.tanita.com/en/technicalbulletin/

19. Mexican Oficial Norm NOM-043-SSA2-2012, Health Services. Promotion and Health Education in Alimentary Matters. Criteria for guidance. Diario Oficial de la Federación., Ciudad de México, 2012.

20. World Health Organization. Obesity: preventing and managing the global epidemic. Report of a WHO Consultation. Geneva: WHO; 2000. Techical Report Series 894.

21. Schlussel MM, Anjos LA, Vasconcellos MT, Kac G. Reference values of handgrip dynamometry of healthy adults: a population-based study. Clin Nutr. 2008; 27: 601-607.

22. Lázaro ML, Lázaro MA, Losantos BF, Plaza A. New tables on hand strength in the adult population from Teruel. Nutr Hosp. 2008; 23: 35-40.

23. Understanding your measurements. United States of America: Tanita Corporation for America Inc.; 2019 [accesed July 18, 2019 J]. Available from: https://www. tanita.com/en/understanding-your-measurements/.

24. Liao KH. Experimental study on gender differences in hands and sequence of force application on grip and hand-grip control. Int J Occup Saf Ergon. 2014; 20: 77-90.

25. Chandrasekaran B, Ghosh A, Prasad C, Krishnan K, Chandrasharma B. Age and Anthropometric Traits Predict Handgrip Strength in Healthy Normals. J Hand Microsurg. 2010; 2: 58-61.

26. Rodríguez-García W, Castañeda L, Orea-Tejeda A, et al. Handgrip strength: Reference values and its relationship with bioimpedance and anthropometric variables. Clin Nutr ESPEN. 2017; 19: 54-58.

27. Meskers CGM, Reijnierse EM, Numans ST, et al. Association of Handgrip Strength and Muscle Mass with Dependency in (Instrumental) Activities of Daily Living in Hospitalized Older Adults -The EMPOWER Study. I Nutr Health Aging. 2019; 23: 232-238.

28. Xu HQ, Shi JP, Shen C, Liu Y, Liu JM, Zheng XY. Sarcopeniarelated features and factors associated with low muscle mass, weak muscle strenght, and reduced function in Chinese rural residents: a cross-sectional study. Arch Osteoporos. 2018; 14.

29. Nakandala P, Manchanayake J, Narampanawa J, Neeraja T, Pavithra S, Mafahir M, et al. Descriptive study of hand grip strength and factors associated with it in a group of young undergraduate students in university of Peradeniya, Sri Lanka who are not participating in regular physical training. Int J Physiother. 2019; 6: 82-88.

30. Al-Asadi JN. Handgrip strength in medical students: Correlation with body mass index and hand dimensions. Asian Journal of Medical Sciences. 2018; 9: 21-26.

31. Das A, Dutta M. Correlation between Body Mass Index and Handgrip Strength and Handgrip Endurance among Young Healthy Adults. J Evid Based Med Healthc. 2015; 2: 3995-4001.

32. Cooper R. Occupational activity across adult life and its association with grip strength. Occup Environ Med. 2016; 73: 425-426. 